\title{
Impacto de la vacunación contra Haemophilus influenzae tipo b en Cuba
}

\author{
Félix O. Dickinson, ${ }^{1}$ Antonio E. Pérez, ${ }^{1}$ Miguel A. Galindo ${ }^{2}$ \\ e Ibrahim Quintana ${ }^{2}$
}

RESUMEN Objetivo. Determinar el impacto de la vacunación de menores de 2 años en Cuba contra Haemophilus influenzae tipo $b$ (Hib), principal agente causal de la meningitis bacteriana en ese país.

Métodos. La disponibilidad de vacunas conjugadas eficaces contra Hib motivó la vacunación nacional en 1999 de niños menores de 2 años, que alcanzó una cobertura de 97\%. El impacto se evaluó mediante el Sistema Nacional de Vigilancia de Meningoencefalitis Bacterianas (SNVMEB).

Resultados. La eficacia global de la vacunación se estimó en $99 \%$ y la incidencia general de la meningoencefalitis por Hib disminuyó de 1,3 a 0,6 por 100000 habitantes (46,1\%), observándose la mayor reducción en niños menores de 5 años (56,1\%). En los menores de 1 año se redujo 70,5\% y en el resto de los grupos de menores de 5 años disminuyó entre 25,9 y 49,6\%. En el grupo diana para la vacunación, la incidencia se redujo 61,1\%; entre los niños de este grupo que contrajeron la meningoencefalitis por Hib, solamente $8(24,2 \%)$ estaban vacunados, 7 de ellos con una sola dosis, aplicada 1 mes antes de enfermar.

Conclusiones. Se ha demostrado que la vacunación a gran escala de los niños menores de 2 años contra Hib en Cuba a través del SNVMEB ha logrado disminuir notablemente la incidencia de meningoencefalitis por Hib.

Palabras clave Haemophilus influenzae tipo b, vacunas, sistema de vigilancia, meningitis bacterianas.

Haemophilus influenzae es una causa importante de meningitis y otras infecciones invasoras en niños menores de 5 años (1). Las cepas encapsuladas de $H$. influenzae del tipo b (Hib) son las más frecuentemente aisladas y pueden llegar a ser las responsables de $95 \%$ de las infecciones causadas por esta bacteria (1).

\footnotetext{
1 Instituto de Medicina Tropical "Pedro Kourín", La Habana, Cuba. Dirección postal: Autopista Novia del Mediodía km. 6, La Lisa, Ciudad de La Habana, Cuba.

2 Ministerio de Salud Pública, La Habana, Cuba.
}

Entre las poblaciones de diferentes países se han observado diferencias en la mortalidad por infecciones causadas por Hib, que puede variar de 15 a 100 defunciones por 100000 habitantes en los países desarrollados (2-4). Después del licenciamiento y uso de las vacunas conjugadas, la incidencia de las meningoencefalitis y de otras infecciones producidas por el Hib disminuyó abruptamente en los países que las han utilizado adecuadamente (5-8), en parte debido al impacto de estas vacunas sobre los portadores nasofaríngeos (9), que ha provocado un cambio impor- tante en la epidemiología de estas infecciones (10).

En los países en vías de desarrollo la incidencia es difícil de calcular con exactitud, pero parece ser elevada. Además, en esos países, en comparación con los países desarrollados, la enfermedad tiene un comienzo más precoz y la letalidad es más alta (1).

En Cuba, Hib es el principal patógeno causante de meningoencefalitis bacteriana (MEB) y el grupo de edad más afectado es el de niños menores de 5 años, y entre ellos el de menores de 1 año. Entre 1994 y 1998 se notificó 
un promedio anual de 59 casos en niños menores de 1 año y 64 casos en niños de 1 a 4 años, con tasas de incidencia que fluctuaron entre 32,2 y 44,0 por 100000 habitantes y 7,2 y 12,9 por 100000 habitantes, respectivamente. Tomando en cuenta estos datos, la frecuencia de otras infecciones por Hib, así como sus secuelas y aporte a la mortalidad infantil, el Ministerio de Salud Pública de Cuba (MINSAP) decidió aplicar una vacuna conjugada contra Hib a través del Programa Nacional de Inmunización (PNI), con el objetivo de disminuir la morbilidad y mortalidad por este agente en los grupos de edad en mayor riesgo $(11,12)$.

Por otra parte, los sistemas de vigilancia permiten proveer información lo más exacta posible sobre los agentes patógenos, la población en riesgo, la letalidad y otros elementos que resultan indispensables para la planificación y ejecución de medidas de intervención beneficiosas para la salud pública.

En Cuba, las MEB se han considerado enfermedades de declaración obligatoria desde 1961, y en los informes estadísticos se consigna el agente causante cuando se identifica; en caso contrario, se notifica como un caso de meningoencefalitis bacteriana sin aislamiento, cuya proporción en los últimos años ha fluctuado entre 40 y 50\% del total de casos. El perfeccionamiento progresivo de este sistema de vigilancia para las meningoencefalitis, basado en experiencias autóctonas (13), permitió implementar en 1998 el Sistema Nacional de Vigilancia de las Meningoencefalitis Bacterianas (SNVMEB), con una nueva perspectiva, caracterizada por la extensión y profundización de la información sobre todos los agentes etiológicos a escala nacional, como parte del Programa Nacional de Prevención y Control de los Síndromes Neurológicos Infecciosos (14), lo que posibilita la evaluación del impacto de las intervenciones con la precisión y oportunidad requeridas.

En el presente trabajo se exponen los resultados de la aplicación por primera vez en Cuba, en 1999, de una vacuna contra Hib en gran escala y su impacto, evaluado a través del SNVMEB, implementado un año antes.

\section{MATERIALES Y MÉTODOS}

\section{Sistema de vigilancia}

El sistema de vigilancia estuvo basado en la información automatizada de la encuesta epidemiológica nacional que se aplica a todos los casos confirmados, la cual forma parte del SNVMEB y es enviada regularmente por cada provincia por correo electrónico al Instituto de Medicina Tropical "Pedro Kourí" (IPK). Se trata de un formulario previamente estandarizado que, además de los datos demográficos, recoge información sobre la atención médica, los factores de riesgo, la vacunación y otros datos microbiológicos y epidemiológicos de importancia para el control focal de cada caso notificado. Para evitar sesgos, los epidemiólogos y el personal estadístico que participaron en la vigilancia fueron adiestrados previamente en cómo llenar dichos formularios.

Las MEB son enfermedades muy graves y ponen en riesgo la vida, por lo que los enfermos habitualmente requieren ingreso hospitalario. Además del Laboratorio Nacional de Referencia del IPK, encargado de la vigilancia microbiológica de todo el país, todos los hospitales disponen de laboratorios donde es factible realizar el diagnóstico etiológico de estas infecciones. Por otra parte, el sistema de salud cubano es estatal y de libre acceso para toda la población, con la asistencia médica y social gratuitas. Estas condiciones y la necesidad de ingreso hospitalario evitan la pérdida de casos a los efectos de la vigilancia y permitió que estas instituciones constituyeran la fuente primaria de notificación de casos para el presente estudio.

La población de Cuba, compuesta de alrededor de 11 millones de personas, estuvo sujeta a la vigilancia entre el primero de enero de 1998 y el 31 de diciembre de 1999, período durante el cual el SNVMEB notificó un total de 209 casos de meningoencefalitis por Hib.Se definió como caso de meningoencefalitis por Hib la presencia de un síndrome clínico compatible con meningoencefalitis, confirmado por un cultivo positivo a Hib o una prueba positiva de aglutinación con látex de líquido cefalorraquídeo o sangre (14).

\section{Vacunación contra Hib}

La estrategia empleada por el MINSAP consistió en vacunar durante 1999 a todos los nacidos entre enero de 1998 y octubre de 1999 (306 098 niños). Para su vacunación se decidió seguir de forma simultánea dos tácticas diferentes (campaña y programa). Para la campaña se estimó un total de 124383 niños (nacidos entre enero y octubre de 1998), seguida por la vacunación de rutina del PNI que incluía a 181715 niños (nacidos vivos entre noviembre de 1998 y octubre de 1999). La vacunación comenzó a efectuarse en la población seleccionada el 1 de enero de 1999 (12).

La vacuna contra Hib Vaxem-Hib ${ }^{\circledR}$ (Chiron Vaccines S.p.A., Italia) está compuesta por oligosacáridos capsulares de la bacteria, conjugados con una proteína portadora denominada CRM 197 (del inglés cross reacting material 197), que es una proteína no tóxica obtenida a partir de una cepa mutante productora de toxina diftérica. Cada dosis de $0,5 \mathrm{~mL}$ contiene $10 \mu \mathrm{g}$ de oligosacárido capsular de Hib conjugado con aproximadamente $25 \mu \mathrm{g}$ de CRM 197. El excipiente se compone de $1 \mathrm{mg}$ de hidróxido de aluminio y 0,05 mg de timerosal.

La vacuna se administró por vía intramuscular en el tercio medio de la cara anterolateral del muslo. En el cuadro 1 se muestran los tres esquemas de vacunación utilizados, de 3, 2 y 1 dosis, con un refuerzo (9).

\section{Análisis de los datos}

Para el cálculo de la incidencia por grupo de edad se utilizó la información sobre la población cubana estimada para 1998 y 1999, disponible en la Oficina Nacional Estadística.

La eficacia de la vacuna (EV) se calculó mediante la fórmula descrita por Orestein y colaboradores (15): EV $=[(\mathrm{TNV}-$ $\mathrm{TV}) / \mathrm{TNV}] \times 100$, donde TNV es la tasa de incidencia acumulada entre el primero de enero de 1998 y el 31 de diciem- 


\section{CUADRO 1. Esquema de vacunación con $\operatorname{Vaxem}^{\circledR}$ en niños de 1 año 0 menores. Cuba, 1999}

\begin{tabular}{ccc}
\hline $\begin{array}{c}\text { Edad a la 1. }{ }^{\text {a dosis }} \\
\text { (en meses) }\end{array}$ & Dosis aplicadas & $\begin{array}{c}\text { Edad en que se } \\
\text { administró el refuerzo } \\
\text { (en meses) }\end{array}$ \\
\hline 12 & 1 & 14 \\
$7-11$ & $0-1$ & 18 \\
$3-6$ & $0-1-2$ & 15 \\
2 & $0-1-2$ & 15 \\
\hline
\end{tabular}

Fuente: Referencia 12.

${ }^{\text {a }}$ Dos meses de intervalo entre dosis.

bre de 1999 en los no vacunados, y TV es la tasa de incidencia acumulada en el mismo período en personas vacunadas.

El análisis de los datos se efectuó mediante el programa Epi Info versión 6.04a (16).

\section{RESULTADOS}

Como consecuencia de la estrategia aplicada se vacunaron contra Hib 296916 niños menores de 2 años durante 1999, obteniéndose una cobertura de vacunación nacional de $97 \%$. La eficacia de la vacunación se estimó en $99 \%$ (intervalo de confianza: 97,8 a 99,6\%) y el riesgo relativo de enfermar entre los vacunados fue 0,01 .

Se apreció una disminución considerable $(52,8 \%)$ del total de casos de meningoencefalitis por Hib en la población bajo vigilancia después de la vacunación masiva de menores de 2 años (de 142 casos en el año 1998, a 67 en 1999), lo que también representó una disminución importante de la incidencia general (de 1,3 a 0,6 por 100000 habitantes).

Por grupos de edad, el mayor número de casos se observó en los menores de 5 años; en mayores de esta edad se notificaron pocos casos (15 casos en 1998 y 11 en 1999) y la incidencia resultó inferior a 0,7 por 100000 habitantes. Desde 1994 se observaba un sostenido incremento anual del número de casos y de la incidencia en menores de 5 años. Al comparar el número de casos en 1998 y 1999 (año en que se comenzó a aplicar la vacuna) se observó una disminución importante del mismo (de 127 a 56) y de la incidencia (de 17,3 a 7,6 por 100000 habitantes) (figura 1).
La mayor reducción se observó en menores de 1 año (70,5\%). Para cada grupo mayor de esa edad la reducción fue disminuyendo progresivamente hasta alcanzar $25.9 \%$ en los niños de 4 años de edad (cuadro 2). En el grupo seleccionado para la vacunación (niños menores de 2 años), los casos disminuyeron de 93 en 1998 a 36 en 1999, lo cual representa una reducción de la incidencia de $61,1 \%$ (30,9 contra 12,0 por 100000 habitantes). La enfermedad afectó de manera similar a ambos sexos, y no se notificó ningún caso secundario o asociado con el caso índice.

De 36 niños menores de 2 años reportados con meningoencefalitis por Hib, 15 eran menores de 1 año (aunque mayores de 2 meses de edad) y 21 teningoencefalitis Bacteriana. nían 1 año de edad cumplido en el momento de enfermar. La información acerca de las dosis de la vacuna aplicadas se obtuvo en 33 casos $(91,6 \%)$. De ellos solo estaban vacunados $8(24,2 \%)$, 7 con una sola dosis de la vacuna y 1 con dos dosis. El intervalo de tiempo más frecuentemente observado entre la vacunación y el comienzo de los síntomas fue de alrededor de 1 mes, fundamentalmente en los niños de 1 año de edad. Un total de 25 niños $(75,8 \%)$ estaban sin vacunar en el momento de enfermar (datos no mostrados).

\section{DISCUSIÓN}

La existencia de un sistema de vigilancia para las MEB permite contar con una importante información epidemiológica sobre los agentes patógenos, la población sometida a un riesgo particularmente alto de contraer la enfermedad, las tendencias de la resistencia antimicrobiana y otros elementos que resultan indispensables en la planificación de medidas para proteger la salud de la población (17). Lamentablemente, en los albores del tercer milenio solo los países desarrollados y algunos en vías de desarrollo disponen de sistemas

FIGURA 1. Casos e incidencia (por 100000 habitantes) de meningoencefalitis por Hib en niño menores de 5 años, por año. Cuba, 1994-1999

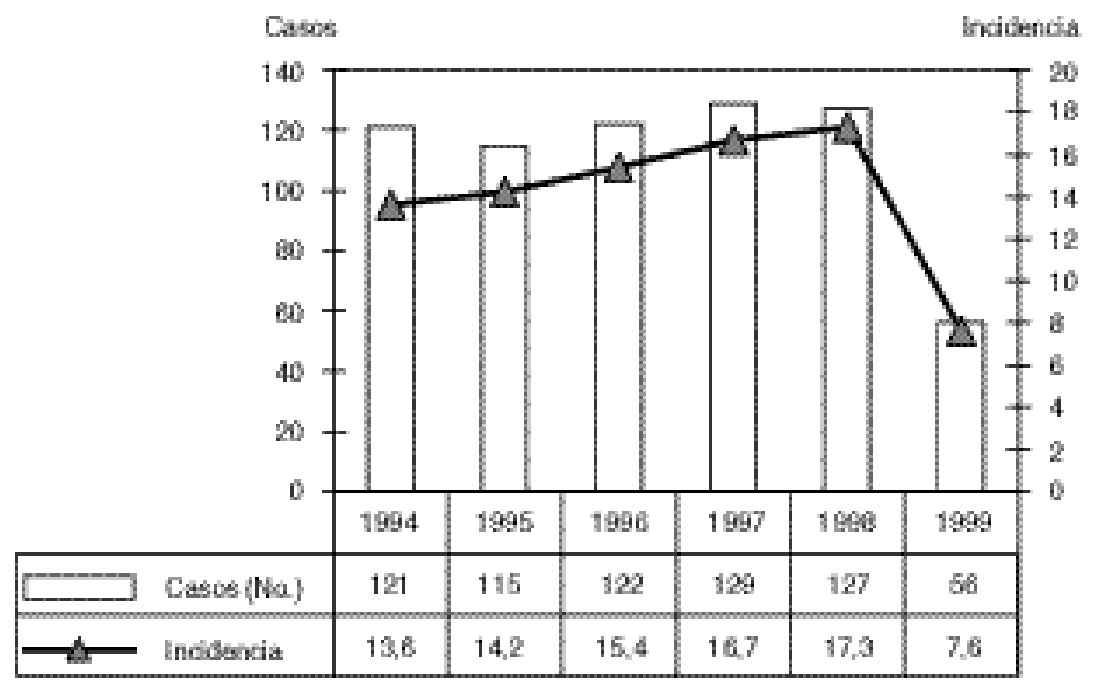

Fuente: Dirección Nacional de Estadísticas del Ministerio de Salud Pública de Cuba y Sistema Nacional de Vigilancia de Me- 
CUADRO 2. Casos de meningoencefalitis por Hib en menores de 5 años, población en cada grupo de edad, incidencia por 100000 habitantes, y porcentaje de reducción de la incidencia (RI), según edad y año. Cuba, 1998-1999

\begin{tabular}{|c|c|c|c|c|c|c|c|}
\hline \multirow{2}{*}{$\begin{array}{l}\text { Edad } \\
\text { (años) }\end{array}$} & \multicolumn{3}{|c|}{1998} & \multicolumn{3}{|c|}{1999} & \multirow[b]{2}{*}{ RI (\%) } \\
\hline & Casos & Población & Incidencia & Casos & Población & Incidencia & \\
\hline$<1$ & 51 & 150190 & 33,9 & 15 & 149978 & 10,0 & 70,5 \\
\hline 1 & 42 & 151127 & 27,8 & 21 & 149734 & 14,0 & 49,6 \\
\hline 2 & 22 & 140499 & 15,6 & 13 & 152428 & 8,5 & 45,5 \\
\hline 3 & 8 & 146869 & 5,4 & 4 & 140399 & 2,8 & 48,1 \\
\hline 4 & 4 & 146448 & 2,7 & 3 & 146717 & 2,0 & 25,9 \\
\hline
\end{tabular}

Fuente: Sistema Nacional de Vigilancia de Meningoencefalitis Bacteriana.

para la vigilancia de estas infecciones $(18,19)$.

La disminución a menos de la mitad de las meningoencefalitis causadas por Hib observada en Cuba se esperaba por varias razones: la experiencia y los buenos resultados del uso de vacunas conjugadas contra el Hib en diversos países del mundo, donde también han provocado una marcada reducción de la incidencia de meningoencefalitis por Hib entre niños menores de 5 años de edad $(2-8,18$, 20-22); la alta eficacia que han demostrado estas vacunas, que en nuestro estudio se estimó en 99\%; y la alta cobertura de vacunación alcanzada en la población menor de 2 años (97\%), que fue la seleccionada para la intervención en todo el país.

La vigilancia de casos permitió demostrar el impacto de la vacunación en este grupo. En los niños entre 2 y 4 años, se observó una menor reducción de la incidencia de meningoencefalitis por Hib, quizá por no haber sido vacunados.

La población de 5 y más años de edad mostró una incidencia baja de la enfermedad, probablemente debido a la inmunidad natural o como efecto de la inmunidad colectiva (herd immunity). Los pocos casos observados po- drían estar relacionados con factores individuales y algunas condiciones socioambientales riesgosas.

Los portadores asintomáticos han sido reconocidos como la mayor fuente de infección (23). De ahí que su reducción justifique la vacunación de personas en grupos de alto riesgo. La tasa de portadores de Hib entre niños saludables es baja en menores de 6 meses, pero se incrementa a partir de esa edad (10). Como la estrategia contra la infección por Hib aplicada en Cuba comprende la vacunación rutinaria de menores de 1 año a partir de 1999 como parte del PNI, la reducción de la tasa de portadores en las cohortes vacunadas y sus efectos en la comunidad se podrán evaluar en un futuro cercano.

En niños de países en desarrollo, Hib es una de las causas principales de infecciones bacterianas graves y causa alrededor de $30 \%$ de los casos de neumonía con cultivo de esputo positivo y $20-60 \%$ de los casos de MEB (24). Otras infecciones causadas por Hib pueden llegar a la mitad de la frecuencia de las meningoencefalitis $(1,25$, 26). De acuerdo a estos datos, la vacunación aplicada en Cuba puede haber prevenido alrededor de 200 casos de la enfermedad invasora, 40 defunciones y 30 casos infantiles con secuelas.
La meningoencefalitis por Hib ocurrió con mayor frecuencia entre los niños no vacunados menores de 2 años de edad, y la mayoría de los casos que ocurrieron en niños vacunados solo tenían 1 dosis aplicada, por lo que estos niños no estaban totalmente protegidos contra la enfermedad (18). No se notificaron casos entre los niños que habían completado su serie primaria de vacunación. Todo esto evidencia que las fallas de la vacuna no son frecuentes cuando se aplica el esquema correctamente (27).

Durante el período de estudio no se notificaron casos secundarios ni asociados con el caso índice de meningoencefalitis por Hib. En Cuba, el Programa Nacional de Prevención y Control de los Síndromes Neurológicos Infecciosos establece que en las 48 horas después de la notificación de un caso confirmado se inicien las actividades de control focales, que abarcan tareas fundamentales, como la educación sanitaria y la vigilancia personal durante 10 días de todos los contactos, y la quimioprofilaxis, aplicable después de un adecuado análisis epidemiológico del caso, a todos los contactos domiciliarios o institucionales donde haya otros niños menores de 5 años. El medicamento de elección es la rifampicina, administrada bajo control del médico o la enfermera en dosis diarias de $10-20 \mathrm{mg} / \mathrm{kg}$ de peso corporal durante 4 días. Además, se orientan medidas generales, como reducir el hacinamiento y mejorar la ventilación domiciliaria e ingresar en el hospital a todo caso sospechoso.

Nuestros datos confirmaron los beneficios de la estrategia de vacunación aplicada en Cuba, y las posibilidades que pueden brindar los sistemas de vigilancia nacionales para la evaluación y el seguimiento de este tipo de estrategias.

\section{REFERENCIAS}

1. Guérin N. Controle des infections à Haemophilus influenzae. Cahiers Santé 1994;4: 227-230.

2. Centers for Disease Control. Haemophilus b conjugate vaccines for prevention of Haemo- philus influenzae type $\mathrm{b}$ disease among infants and children two months of age and older: Recommendations of Immunization Practices Advisory Committee (ACIP). MMWR 1991; 40:1-7.
3. Macleod CA. Haemophilus influenzae: the efficiency of reporting invasive diseases in England and Wales. CDR Review 1994;4:13-16.

4. Peltola H, Kilpi T, Anttila M. Rapid disappearance of Haemophilus influenzae type $\mathrm{b}$ menin- 
gitis after routine childhood immunization with conjugate vaccines. Lancet 1992;340: 592-594.

5. Adams WG, Deaver KA, Cochi SL, Plikaytis $\mathrm{BE}$, Bell ER, Broome CV, et al. Decline of childhood Haemophilus influenzae type $\mathrm{b}$ (Hib) in the Hib vaccine era. JAMA 1993;269: 221-226.

6. Agudelo CI, Muñoz N, De la Hoz F y Laboratorios de Salud Pública. Evaluación rápida del impacto de la vacuna contra Haemophilus influenzae serotipo b en Colombia. Rev Panam Salud Publica/Pan Am J Public Health 2000; 8:181-184.

7. Ruocco G, Curto S, Savio M, Laurani H, Froch R. Vacunación contra Haemophilus influenzae tipo b en el Uruguay: experiencia e impacto. Rev Panam Salud Publica/Pan Am J Public Health 1999;5:197-199.

8. Landaverde M, Di Fabio JL, Ruocco G, Leal I, de Quadros C. Introducción de la vacuna conjugada contra Hib en Chile y Uruguay. Rev Panam Salud Publica/Pan Am J Public Health 1999;5:200-206.

9. Takala AK, Eskola J, Leinonen M, Kaythy H, Nissinen A, Pekkanen, et al. Reduction of oropharyngeal carriage of Haemophilus influenzae type $\mathrm{b}$ (Hib) in children immunised with an Hib conjugate vaccine. J Infect Dis 1991;164:982-986.

10. Schuchat A, Robinson K, Wenger JD, Harrison LH, Farley M, Reingold A, et al. Bacterial meningitis in the United States in 1995. N Engl J Med 1997;337:970-976.

11. Tamargo I, Toraño G, Fuentes K, Rodríguez O. Haemophilus influenzae circulantes en Cuba. Caracterización de cepas aisladas de meningitis. Rev Cub Med Trop 1999;51:204-205.

12. Cuba, Dirección Nacional de Epidemiología. Programa de Vacunación contra el Haemophilus influenzae en la República de Cuba. Ciudad de La Habana: Ministerio de Salud Pública; 1998.
13. Pérez AE, Dickinson FO, Baly A, Martínez R. The epidemiological impact of antimeningococcal B vaccination in Cuba. Mem Inst Oswaldo Cruz 1999;94:433-440.

14. Cuba, Dirección Nacional de Epidemiología. Programa Nacional de Prevención y Control de los Síndromes Neurológicos Infecciosos. Ciudad de La Habana: Ministerio de Salud Pública; 1999.

15. Orestein WA, Bernier RH, Alan BH. Assessing vaccine efficacy in the field: further observations. Epidemiol Rev 1988;10:212-240.

16. Dean AG, Dean JA, Coulombier D, Brendel KA, Smith DC, Burton AH, et al. Epi Info, Version 6.04a: A word processing, database, and statistics program for public health on IBMcompatible microcomputers. Atlanta: Centers for Disease Control and Prevention; 1996.

17. Wenger JD, Hightower AW, Facklam RR, Gaventa $S$, Broome $C V$ y Bacterial Meningitis Study Group. Bacterial meningitis in the United States, 1986: report of a multistate surveillance study. J Infect Dis 1990;162: 1316-1323.

18. Bisgard KM, Kao A, Leake J, Sterbel PM, Perkins BA, Wharton M. Haemophilus influenzae invasive disease in the United States, 1994-1995: near disappearance of a vaccinepreventable childhood disease. Emerg Infect Dis 1998:4:229-238.

19. John TJ, Samuel R, Balraj V, John R. Disease surveillance at district level: a model for developing countries. Lancet 1998;352:58-61.

20. Centers for Disease Control and Prevention. Progress toward elimination of Haemophilus influenzae type $\mathrm{b}$ disease among infants and children. United States 1987-1995. MMWR 1996;45:901-906.

21. Takala AK, Peltola H, Eskola J. Disappearance of epiglottitis during large-scale vaccination with Haemophilus influenzae type b conjugated vaccines among children in Finland. En: Abstracts of the 11th Meeting of the European So- ciety for Pediatric Infectious Diseases (ESPID); May 26-28 1993; Helsinki, Finland; 1993. Abstract 67.

22. Centers for Disease Control and Prevention. Reported vaccine-preventable disease -United States, 1993, and the Childhood Immunization Initiative. MMWR 1994;43:57-60.

23. Barbour ML, Phil D. Conjugated vaccines and the carriage of Haemophilus influenzae type b. Emerg Infect Dis 1996;2:176-182.

24. Funkhouser A, Steinhoff MC, Ward J. Haemophilus influenzae disease and immunization in developing countries. Rev Infect Dis 1991; 13(Supl. 6):S542-S554.

25. Granoff DM, Basden M. Infections in Fresno County, California: a prospective study of the effects of age, race, and contact with a case on incidence of disease. J Infect Dis 1980;141: 40-46.

26. Takala AK, Eskola J, van Alpen L. Spectrum of invasive Haemophilus influenzae type b disease in adults. Ann Intern Med 1990;150: 2573-2576.

27. Jafari H, Adams W, Deaver K, Plikaytis B, Wenger J. Efficacy of Haemophilus influenzae type $\mathrm{b}$ (Hib) conjugated vaccine, risk factors for invasive Hib disease and under-vaccination in the United States. En: Abstracts of the Interscience Conference on Antimicrobial Agents and Chemotherapy, 1995, San Francisco, California. Washington, D.C.: American Society for Microbiology; 1995. pp. 160.

Manuscrito recibido el 23 de febrero de 2001 y aceptado para publicación, tras revisión, el 31 de mayo de 2001.

ABSTRACT Objective. To assess the effectiveness of mass vaccination in Cuba of children under 2 years of age against Haemophilus influenzae type b (Hib), the most common causative pathogen of bacterial meningitis.

Impact of vaccination against Haemophilus influenzae type $b$ in Cuba

Methods. The availability of effective Hib conjugate vaccines led to a nationwide vaccination program in 1999 targeting all children under 2 years of age, with a 97\% coverage rate achieved. To assess the program's impact, data from 1998 and 1999 from the National Bacterial Meningitis Reporting System were used.

Results. Vaccination efficacy was estimated at $99 \%$. The overall incidence of Hib meningitis declined $46.1 \%$, from 1.3 to 0.6 cases per 100000 population. The greatest overall reduction, of $56.1 \%$, occurred among children under 5 years of age. Among children under 1 year of age, the reduction was $70.5 \%$, and among the rest of the age groups of children under 5, incidence decreased between $25.9 \%$ and $49.6 \%$. In the group targeted for vaccination, incidence decreased $61.1 \%$. Among children in the target group who contracted Hib meningitis, only 8 cases $(24.2 \%)$ had been vaccinated, most with a single dose applied 1 month before becoming ill.

Conclusions. Hib vaccination of all children under 2 years of age in Cuba greatly reduced the incidence of Hib meningitis, as measured by the National Bacterial Meningitis Reporting System. 